\title{
Real-time measurement of oxidation dynamics of sub-stoichiometric tungsten oxide films by pulsed laser deposition
}

\author{
Mehdi Ranjbar • Azam Iraji zad • Seyyed Mohammad Mahdavi
}

Received: 8 April 2008 / Accepted: 17 April 2008 / Published online: 7 June 2008

(C) The Author(s) 2008

\begin{abstract}
In this study $\mathrm{WO}_{x}$ films were deposited by laser ablation of ultra-pure $(5 \mathrm{~N})$ tungsten trioxide targets onto $\mathrm{SiO}_{2}$ or silicon substrates at $250^{\circ} \mathrm{C}$ temperature, $100 \mathrm{mTorr}$ oxygen partial pressure and $1 \times 10^{-5}$ Torr vacuum. Surface chemical states and compositions of the deposits were determined by X-ray photoelectron spectroscopy. The results showed that deposits in oxygen partial pressure contain $\mathrm{W}^{6+}$ with $x \sim 3.1$, while vacuum-deposited films have different $\mathrm{W}$ states with various percentage distributions as $\mathrm{W}^{4+}>\mathrm{W}^{5+}>\mathrm{W}^{6+}>\mathrm{W}^{0}$, and $x \sim 1$. We used fast electrical resistance measurement as a probe to study the deposition process. Film resistance as a function of deposition time in vacuum revealed some microsecond fluctuations modulated on the time variation curve of electrical resistance. We attribute these data to surface absorption and desorption of oxygen during layer deposition. Finally, the effect of the laser beam on the target's structure, surface morphology and chemical state was studied. Our results revealed that in spite of structural variation by laser irradiation, the $\mathrm{O} / \mathrm{W}$ ratio remained about 3 .
\end{abstract}

PACS 68.47.Gh $\cdot$ 77.84. Bw $\cdot$ 68.43.Mn $\cdot$ 73.61.Jc 79.20.Ds

\author{
M. Ranjbar · A. Iraji zad ( $\varangle) \cdot$ S.M. Mahdavi \\ Department of Physics, Sharif University of Technology, Azadi \\ Av., Tehran, Iran \\ e-mail: iraji@sharif.edu \\ M. Ranjbar \\ e-mail: mranjbar@physics.sharif.edu \\ S.M. Mahdavi \\ e-mail: mahdavii@sharif.edu \\ A. Iraji zad · S.M. Mahdavi \\ The Institute for Nanoscience and Nanotechnology (INST), Sharif \\ University of Technology, P.O. Box 11365-9161, Tehran, Iran
}

\section{Introduction}

$\mathrm{WO}_{x}$ (tungsten oxide) is a widely studied metal oxide semiconductor material and has very interesting technological applications in electronics and optoelectronics industries. It is used as gas sensors, super windows, optical storage devices and displays [1]. Up to now several investigated deposition methods were applied to fabricate tungsten oxide films on different substrates. Among them pulsed laser deposition (PLD) is recognized as a successful deposition technique to fabricate qualify tungsten oxide thin films for different applications [2-4]. In the case of metal oxides, this technique is usually accompanied with oxygen to produce stoichiometric $\mathrm{WO}_{3}$ films but, in low-pressure deposition, the composition of the films is expected to be $\mathrm{WO}_{x<3}$ and may also have a metallic nature [4]. In the PLD method, ablation of a composite target in vacuum condition is a complex process and, to the best of our knowledge, sub-stoichiometric deposition by ablation of a stoichiometric $\mathrm{WO}_{3}$ target has not been completely understood. Since the lack of oxygen may be related to the escape of oxygen molecules from the incident plume to the substrate and/or the deposit's surface, study of both cases is of benefit.

One characteristic of metal oxide films that strongly depends on oxygen level in the layer is electrical resistance. So, during film growth we can use electrical resistance measurement as an approach to detect oxygen surface absorption or desorption. This method has been used to study growth mechanisms or gas-surface interactions [4, 5].

The thickness-dependent electrical resistance of a film usually exhibits a fast drop at the percolation threshold, which is followed by a reducing trend with lower rate [6]. This behavior can be observed in long-time-scale resistance measurements. However, measurement of electrical resistance at short time scales may reveal fast phenomena 
in the film-growth mechanism similar to what is observed in plasma processes by optical spectroscopy. Nanosecond pulsed laser irradiation of the target surface indicates a complex sequence of events which occurs both during and after impinging of the laser pulse. These include laser absorption at the surface, phase change and mass expulsion in both liquid and vapor states, plasma formation leading to partial absorption in the plume, accelerating of the vapor to hypersonic velocities followed by rapid condensation and finally collapse of the plume onto the substrate surface [7]. The investigations of the plasma plume are generally performed by various optical techniques such as mass spectroscopy and probe methods [8]. The probe can be as simple as wire tips or small electrodes that are immersed directly into the plasma. A power supply provides a bias voltage to the probe and the collected current is displayed on an oscilloscope. In this paper, with the help of the probe method, we studied sub-stoichiometry of tungsten oxide films. Our probe consists of a double thin Au contact on $\mathrm{SiO}_{2}$ substrates placed perpendicular to the plume expansion direction. At the initial stages of growth it works similarly to a Langmuir probe [8]; however, after formation of a conducting network on the substrate surface, current signals that pass through the layer could be detected. In the PLD method, depending on the laser repetition rate, there is a time interval between each laser shot (each deposition step) where the substrate is not bombarded with plume specimens. So, some phenomena such as surface diffusion, desorption or adsorption of species can take place at the deposited layers. We assert that it is possible to monitor these processes by fast measurement of electrical resistance. This method provides a new approach for study of pulsed laser deposited composite films.

\section{Experimental}

The deposition process was run in a vacuum chamber, which was evacuated by a turbo molecular pump down to $1 \times 10^{-5}$ Torr. For deposition in oxygen partial pressure, pure $\mathrm{O}_{2}$ gas (99.999\%), was used and the pressure was kept at 100 mTorr. Tungsten trioxide targets with $10-\mathrm{mm}$ diameter and 5-mm thickness were prepared by pressing ultrapure tungsten trioxide $(5 \mathrm{~N})$ powder. These were then sintered at $900^{\circ} \mathrm{C}$ in air atmosphere for $8 \mathrm{~h}$. Films were deposited by ablating the target using a Q-switched pulsed $\mathrm{Nd}$ :YAG laser (wavelength $1064 \mathrm{~nm}$ and pulse duration of about $10-12 \mathrm{~ns}$ ) operating at $5-\mathrm{Hz}$ repetition rate. The energy density on the target was maintained at $4 \mathrm{~J} / \mathrm{cm}^{2}$. The laser beam was focused at an incident angle of $45^{\circ}$ onto the surface of the target and the distance between the target and substrate was $3 \mathrm{~cm}$. The target was rotated with a speed of $12 \mathrm{rpm}$ during the deposition to minimize texturing of its surface. The substrates were $\mathrm{SiO}_{2}$ or $\mathrm{Si}(111)\left(5 \times 10 \mathrm{~mm}^{2}\right)$ and were cleaned ultrasonically by ethanol and de-ionized water. As our experimental observation indicates, for a desirable layer adhesion to the substrate, a substrate temperature of at least $250^{\circ} \mathrm{C}$ is needed, so all experiments were performed at this temperature. This is due to better surface diffusion of specimens. The deposition rate was measured by a quartz crystal microbalance (QCM) in a separate experiment. Since the distribution of species in the plume is highly localized, simultaneous thickness measurement during film growth is problematical in PLD. Therefore, in order to determine the growth rate correctly, the quartz crystal microbalance was located at the same position of the substrate with the same deposition conditions. Surface analysis of the samples was done in an ESCA/AES system. The system is equipped with a concentric hemispherical analyzer (CHA, Specs model EA10 plus) suitable for Auger electron spectroscopy (AES) and X-ray photoelectron spectroscopy (XPS). For AES measurement, the incident electron beam energy was set to $5 \mathrm{keV}$ and the electron beam current was $10 \mu \mathrm{A}$. The data acquisition was performed at a nominal resolution of $0.1 \mathrm{eV}$. For exciting the X-ray photoelectrons, an $\mathrm{Al} \mathrm{K} \alpha$ line at $1486.6 \mathrm{eV}$ was used. The energy scale was calibrated against the carbon binding energy (284.8 eV). For fast data recording of electrical resistance an oscilloscope (model DS-1250, $250 \mathrm{MHz}$ ) was used. Before ablation, two $\mathrm{Au}$ contacts were coated by thermal evaporation on $\mathrm{SiO}_{2}$ substrates with 5-mm separation distance. For data recording, the sample was connected in series with an $r=22 \mathrm{M} \Omega$ constant resistance. A schematic of the measurement setup is shown in Fig. 1. The deposition chamber was grounded and the circuit remained floating. A DC bias voltage of $10 \mathrm{~V}$ was applied and the film resistance was calculated by a simple relation between two serial resistances. Therefore, any

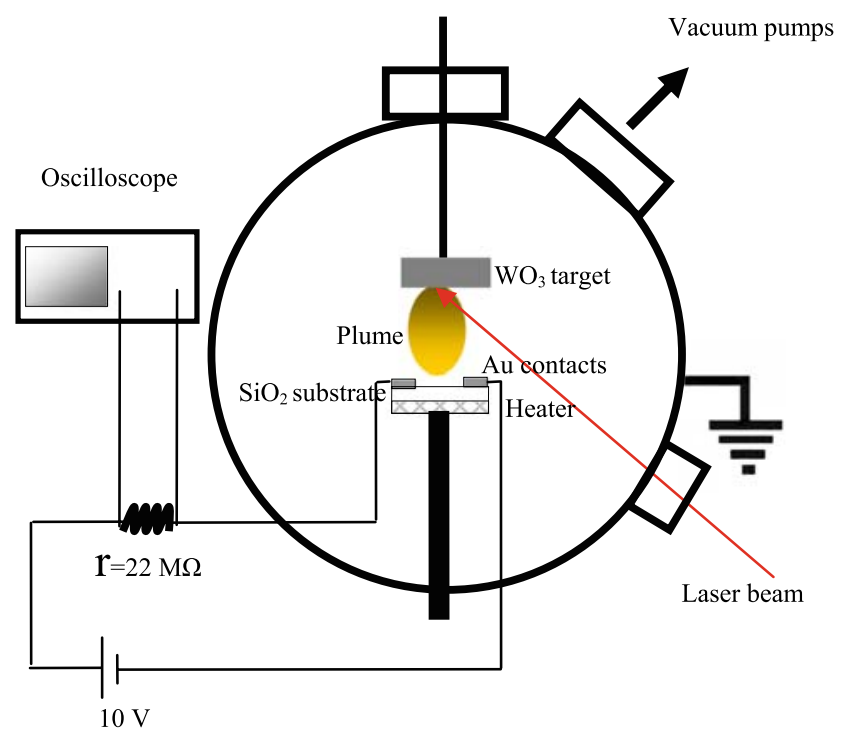

Fig. 1 A schematic of PLD chamber and film resistance measurement setup 


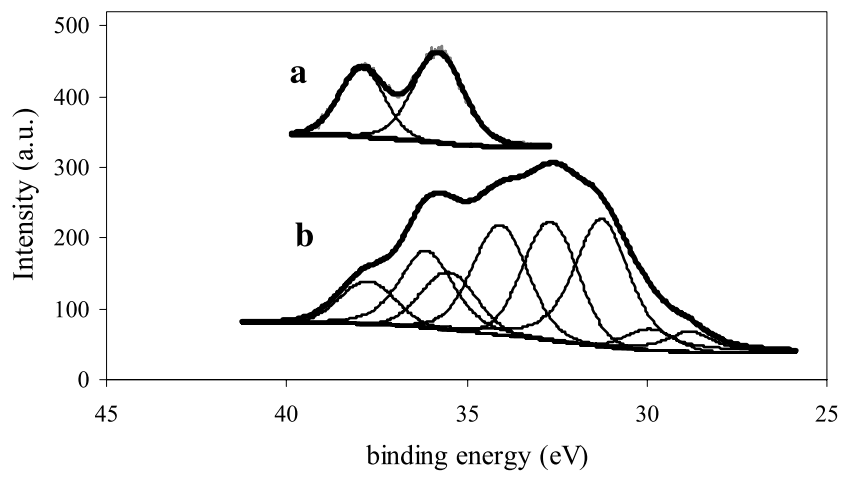

Fig. 2 High-resolution XPS spectra of $\mathrm{W}_{4 f}$ peaks of deposited films in (a) 100 mTorr oxygen partial pressure and (b) $1 \times 10^{-5}$ Torr vacuum condition

change in the sample resistance could be recorded by variation in the voltage of the reference resistance. X-ray diffraction (XRD) measurements were carried out using a Bruker D4 X-ray diffractometer. The $\mathrm{Cu} \mathrm{K} \alpha(1.54 \AA) \mathrm{X}$-ray line was used as the probe beam. Finally, scanning electron microscopy (SEM) characterizations have been achieved using a Philips XL30 electron microscope.

\section{Results and discussion}

\subsection{Deposit composition}

To determine surface chemical compositions we utilized the XPS technique. The shift of core-electron binding energy is the result of charge transfer between $\mathrm{W}$ and $\mathrm{O}$ atoms [9]. The high difference of electro-negativity of the two involved atoms results in different spectra. Therefore, the various oxide states or metallic counterparts of metal oxide films should be clearly distinguishable in XPS. Highresolution XPS spectra of $\mathrm{W}_{4 f}$ transitions of $\mathrm{WO}_{x}$ deposited in 100 mTorr oxygen partial pressure and vacuum condition are shown in Fig. 2. These peaks were fitted using Gaussian and Lorentzian curves after subtraction of a Shirley-type background. Their FWHM ranged from 1.4 to $1.8 \mathrm{eV}$. The doublets on the 35.5 and $37.7 \mathrm{eV}$ are attributed to $\mathrm{W}_{4 f 5 / 2}$ and $\mathrm{W}_{4 f 7 / 2}$, respectively, and are at the same binding energy of tungsten atoms with +6 formal oxidation number as in $\mathrm{WO}_{3}$ [10]. A general agreement exists for the binding energies of $\mathrm{W}^{6+}$ at $35.6 \pm 0.2$ and $37.7 \pm 0.2 \mathrm{eV}$ $[10,11]$. For vacuum-deposited films, the spectrum is a superposition of various peaks corresponding to several $\mathrm{W}$ oxide states. In addition to $\mathrm{W}^{6+}$, two peaks are located at 34.2 and $36.2 \mathrm{eV}$. According to the literature, this doublet is related to $\mathrm{W}^{5+}$ [12], while those peaks at 31.2 and $32.7 \mathrm{eV}$ are attributed to the $\mathrm{W}^{4+}$ state. In spite of $\mathrm{W}^{6+}$, the binding energy related to $\mathrm{W}^{5+}$ and $\mathrm{W}^{4+}$ can vary within $1 \mathrm{eV}[10$, 13-16]. Also, the data show that the film partially contains
Table 1 Binding energy, FWHM and distribution of tungsten states of the samples

\begin{tabular}{|c|c|c|c|c|}
\hline \multirow[t]{2}{*}{ Assignment } & \multicolumn{2}{|c|}{$\begin{array}{l}\text { Deposited in } \\
100 \mathrm{mTorr}_{2}\end{array}$} & \multicolumn{2}{|c|}{ Deposited in vacuum } \\
\hline & $\begin{array}{l}\mathrm{BE} \\
(\mathrm{FWHM})\end{array}$ & $\% \mathrm{~W}$ & $\begin{array}{l}\mathrm{BE} \\
(\mathrm{FWHM})\end{array}$ & $\% \mathrm{~W}$ \\
\hline $\mathrm{W}^{6+}$ & $\begin{array}{l}35.5-37.7 \\
(1.4)-(1.6)\end{array}$ & 100 & $\begin{array}{l}35.5-37.7 \\
(1.8)-(1.8)\end{array}$ & 16 \\
\hline $\mathrm{W}^{5+}$ & - & - & $\begin{array}{l}34.2-36.2 \\
(1.8)-(1.8)\end{array}$ & 33 \\
\hline $\mathrm{W}^{4+}$ & - & - & $\begin{array}{l}31.2-32.7 \\
(1.8)-(1.8)\end{array}$ & 44 \\
\hline $\mathrm{W}^{0}$ & - & - & $\begin{array}{l}28.8-30.0 \\
(1.5)-(1.6)\end{array}$ & 7 \\
\hline
\end{tabular}

metallic tungsten, identified by the two remaining peaks in the spectrum $\left(\mathrm{W}^{0}\right)$. The estimated amounts of surface species percentages calculated using various doublet peak areas are indicated in Table 1.

For the latter case, one can see that the distribution of surface chemical state concentrations is $\mathrm{W}^{4+}>\mathrm{W}^{5+}>\mathrm{W}^{6+}>$ $\mathrm{W}^{0}$. In order to identify the $\mathrm{O} / \mathrm{W}$ ratio, we used the following formula:

$C_{\mathrm{O}} / C_{\mathrm{W}}=\left(\mathrm{PA}_{\mathrm{O}} / S_{\mathrm{O}}\right) /\left(\mathrm{PA}_{\mathrm{W}} / S_{\mathrm{W}}\right)$,

where $C$ is the concentration, PA is the peak area and $S$ is the corresponding sensitivity factor [17]. Quantitative analysis using $\mathrm{O}_{1 s}$ and $\mathrm{W}_{5 d}$ (not shown here) revealed $\mathrm{WO}_{x \sim 3.1}$ and $\mathrm{WO}_{x \sim 1}$ compositions for 100 mTorr oxygen partial pressure and vacuum condition, respectively. In fact, tungsten oxide films are based on corner-sharing $\mathrm{WO}_{6}$ octahedra and it could be assumed as a building block for amorphous in addition to crystalline structures [18]. Each tungsten oxide could be surrounded by six oxygen ions ideally forming an octahedron but, in the sub-stoichiometric films, defects exist mostly in forms of oxygen vacancies and, therefore, lower oxides such as $\mathrm{W}^{5+}$ and $\mathrm{W}^{4+}$ states could be observed. As is mentioned in the introduction, measurements of sample electrical resistance were utilized as a tool for film-growth investigation. XPS data showed that deposited films in oxygen partial pressure are stoichiometric with $\mathrm{W}^{6+}$ state. According to the literature, the Fermi level of $\mathrm{WO}_{3-y}$ progresses into the conduction band for $y \geq 0.2$ because the density of states (DOS) decreases in the valence band. Therefore a metal-insulator transition occurs in sub-stoichiometric tungsten oxide [19]. Therefore, measuring the conductivity of the sample can be used as a method for rough chemical state determination. 

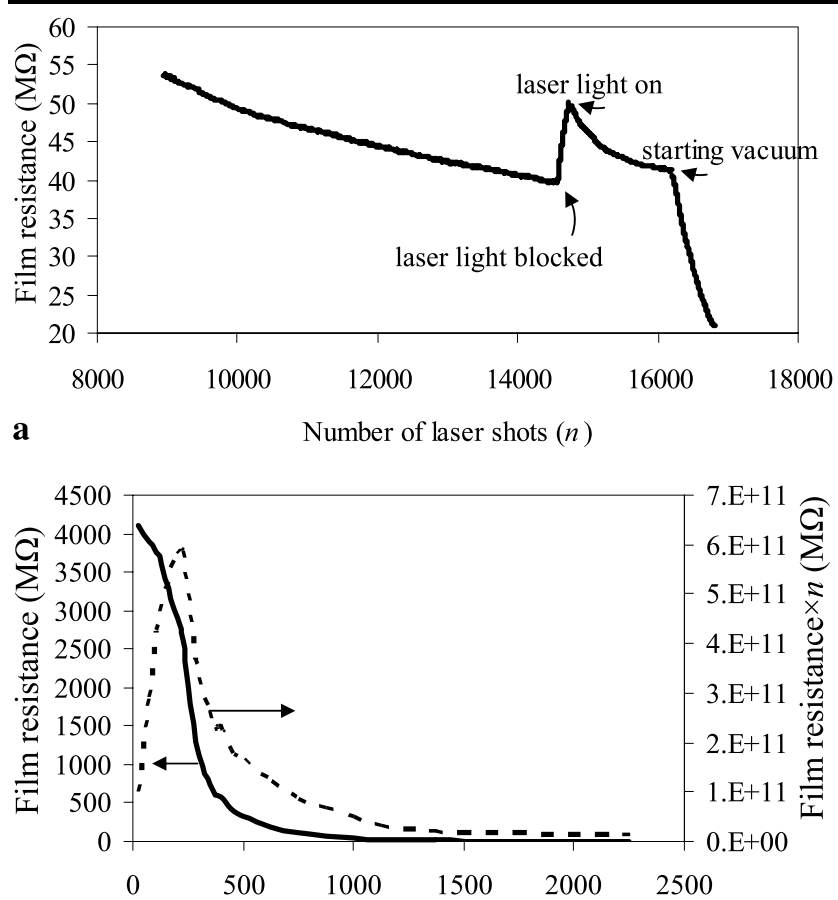

b

Number of laser shots $(n)$

Fig. 3 (a) Film resistance measurement of film deposited in 100 mTorr oxygen partial pressure. (b) Plot of resistance, $R$ (solid line) and $R \times n$ (dashed line) as a function of shot number, $n$

\subsection{Film electrical resistance measurements}

The dependency of film resistance on laser illumination shots, corresponding to different deposition conditions, is presented in Fig. 3. In the case of oxygen partial pressure in Fig. 3a, the film's electrical resistance decays to less than $55 \mathrm{M} \Omega$ by 9,000 or more illumination shots, indicating the possibility of a metal-rich growth mechanism in an oxygen environment by PLD. One can see that the electrical resistance begins to increase when laser irradiation is blocked, but it decays again by subsequent laser exposure. Also, when the chamber is evacuated while deposition is continuing, the film resistance curve represents a faster decay. These results propose a competition process between growth of a metal-rich layer and surface oxidation where, in the present case, the dominant one is metal-rich growth. It seems that although in the presence of oxygen partial pressure stoichiometry is obtainable, the whole oxidation process of the film is completed not during film growth but after being in the oxygen environment for a short period. Nevertheless, it is worth to know how the oxygen counterpart is lost in the lack of oxygen reactive gas. XPS data of vacuum deposits revealed $\mathrm{WO}_{x \sim 1}(y>0.2)$ compositions with lower oxide states and metallic nature. Then, lower and measurable resistance is expected for these samples in comparison to the former ones. Figure $3 \mathrm{~b}$ shows the sample resistance, $R$, and also $R \times n$ versus $n$, where $n$ is the number of laser shots.
Fig. 4 The AES spectra of

$\mathrm{WO}_{x} / \mathrm{Si}(111)$ at distinct steps of deposition in vacuum condition. The doublet Auger peaks located at 168 and $179 \mathrm{eV}$ are related to tungsten and the peak at $85 \mathrm{eV}$ is related to the $\mathrm{Si}$ substrate. The numbers of laser shots, $n$, are 50, 150 and 250

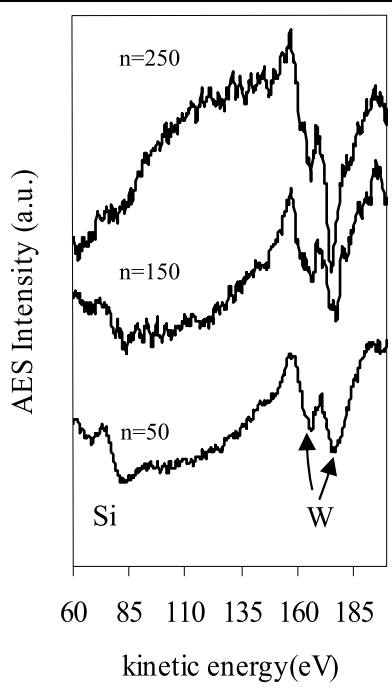

Here, $R$ is the sample resistance calculated from the background voltage curve after $n$ laser shots. We observed some short-time-width fluctuations in voltage signals $\left(V_{\mathrm{r}}\right)$ on the background curve just after the laser shots. This phenomenon was observed only in the vacuum deposition experiment. The quantity $R \times n$ provides us with an estimate of the film percolation threshold, where $n$ is the number of laser shots. At the initial stages of deposition, small islands are formed and then coalescence at the surface of the substrate. Then, they become connected to each other, resulting in reduction of sample resistance. Therefore, we expect to observe rapid resistance variations at the primary stages. Thus, $R \times n$ has a rising shape in this stage. However, at the percolation threshold, it reaches a maximum where the islands are completely connected to each other and form a conducting network. Then, as the number of shots increases, the film resistance decreases considerably. The $R \times n$ curve for our experiments indicates a percolation threshold at $n \sim 225$. For more details we studied the surface chemical composition of tungsten oxide films on a $\mathrm{Si}(111)$ substrate by the AES technique, which were prepared in distinct deposition steps. A silicon substrate was used to avoid the charging effect during electron-beam bombardment in the AES experiment. The samples were prepared in the same condition as described in Sect. 2. Figure 4 shows the differential mode AES spectra of deposited films after 50, 150 and 250 laser shots. W (NVV) doublet Auger peaks are located at 168 and $179 \mathrm{eV}$. The peak at kinetic energy of $85 \mathrm{eV}$ corresponding to the $\mathrm{Si}$ substrate was shifted relative to a typical $\mathrm{Si}^{0}$ Auger transition (at $90 \mathrm{eV}$ ) due to interface chemical bonds. Comparison of the spectra indicates that by increasing the number of laser shots, the Auger intensity of tungsten atoms increases, and also the Si intensity approaches zero for some value of $n$ higher than 250. This result supports the previous result for film electrical resistance measurement, which provides percolation at about $n=225$. 
Fig. 5 Measured voltage of the constant resistance $R$ at different deposition stages

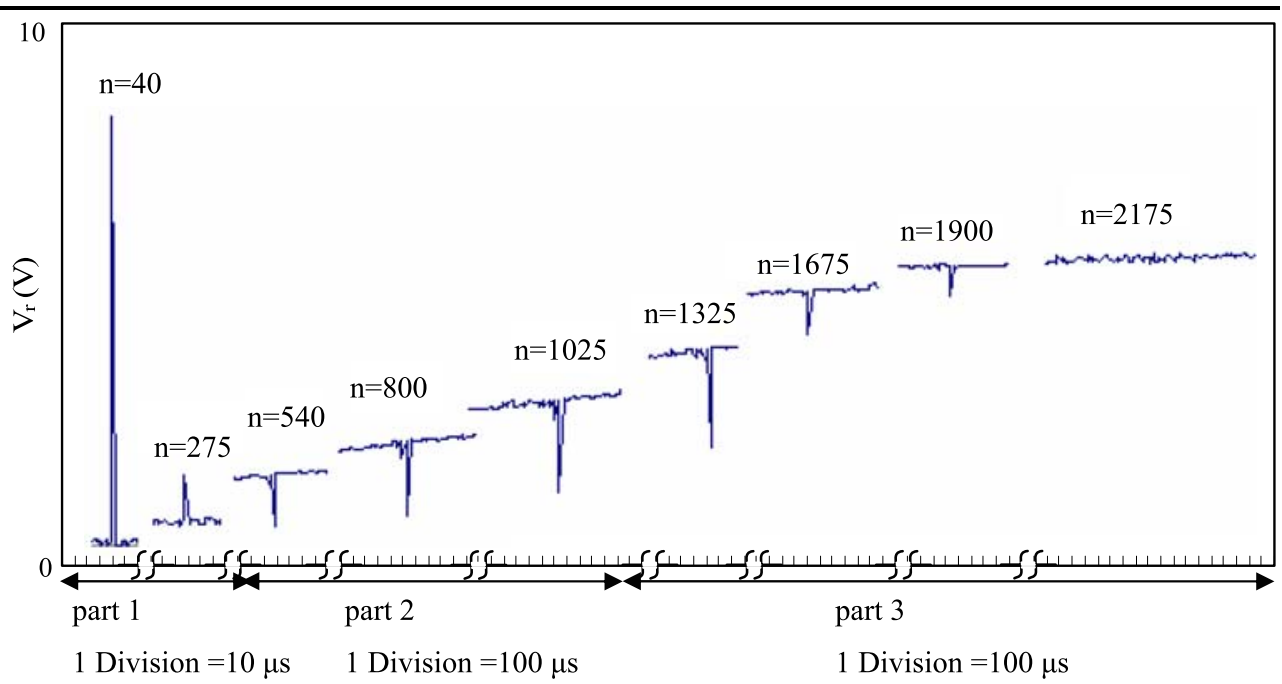

To study the shape and origin of voltage fluctuations, we demonstrate a sequence of some selected instabilities at distinct stages of the deposition process (Fig. 5). Fast data recording using an oscilloscope exhibits microsecond instabilities modulated on the background resistance curve. In fact, the background indicated a smooth increase in sample conductance after percolation as a function of deposition time that we described before. The first upward peak indicates rapid reducing of sample electrical resistance at the initial stage of film growth. Then, the peak height decreases and vanishes after about 275 shots and finally changes to a small downward peak at about 315 pulses (not shown here). By further deposition, the intensity of such peaks increases until about 1,025 shots and then decreases gradually to zero at around 2,175 shots. To understand this behavior we divided the process into three parts. In the first part (upward peaks), including the percolation threshold, plume species are condensed at the substrate and nucleation of islands takes place, but they do not completely coalesce together. The upward voltage peaks across the reference resistance, $V_{\mathrm{r}}$, are mainly due to increase of conductance related to electrical current passing through the plume-electrode contact medium. In fact, before formation of a continuous film, the Au contacts act similarly to the Langmuir probe. By further deposition, the Au contact is covered by a tungsten oxide layer and electrical conductance through the arrival plume reduces. Although the fluctuation intensity is expected to vanish afterwards and remain zero, new downward fluctuation signals appear in the data, representing a sequence of sudden decay-recovery of film conductance. To explain the detail of such fluctuations, we studied the effect of conduction through the plume on the measurements by another experiment that was performed using two individual adjacent Au electrodes with separation distances the same as the previous Au contacts. The measured signal with about $10-\mu$ s width is shown in Fig. 6a. To give an estimate of the time duration of plume-electrode contacts we use the following equation that is based on transfer of thermal energy to kinetic energy during the adiabatic expansion of the plume in vacuum:

$V=\left(\frac{2}{\gamma-1}\right)\left(\frac{\gamma k T}{m}\right)^{1 / 2}$,

where $V$ is the speed of the particles, $\gamma$ is the ratio of specific heats $\left(C_{p} / C_{v}\right), k$ is the Boltzmann constant, $T$ is the temperature and $m$ is the particle mass (see p. 131 of Ref. [9]). Using $\gamma=1.4$ for $\mathrm{WO}_{3}, T=10,000 \mathrm{~K}$ and assuming particles with maximum and minimum masses for our system as $\mathrm{O}(16)$ and $\mathrm{WO}_{3}$ (232), the predicted velocities are about $1.3 \times 10^{6} \mathrm{~cm} / \mathrm{s}$ and $0.34 \times 10^{6} \mathrm{~cm} / \mathrm{s}$ for $\mathrm{O}$ and $\mathrm{WO}_{3}$, respectively. Therefore, considering $3 \mathrm{~cm}$ as target to sample distance, the time of flight for exiting particles, molecules and atoms in the plume should be $\sim 6.5 \mu$ s, which is less than the measured value. Although laser parameters such as pulse length may contribute to deviation of plume length, species of $\mathrm{W}_{x} \mathrm{O}_{y}$ clusters with masses higher than $\mathrm{WO}_{3}$, as reported by a mass spectroscopy study of sublimated $\mathrm{WO}_{3}$ in vacuum conditions [20], could be a factor. So, a similar process is expected for laser ablation of a $\mathrm{WO}_{3}$ target and the observed 10- $\mu$ s width is reasonable due to the existence of large species. In the third part of Fig. 5, the peak height decreases until it vanishes at around 2175 shots when the film thickness is about $108 \mathrm{~nm}$. Figure $6 \mathrm{~b}$ shows the details of the fluctuation signal at nearly 1025 shots where two distinct parts with different time widths are visible. The first part is a sudden dropping of conductance with $\sim 10-\mu$ s time width, which is near the plume-surface contact period (Fig. 6a), and the second part represents an exponential recovery to background at about $100 \mu \mathrm{s}$. With regard to such a feature and remembering that a voltage fluctuation was not observed during the deposition in oxygen partial pressure, 

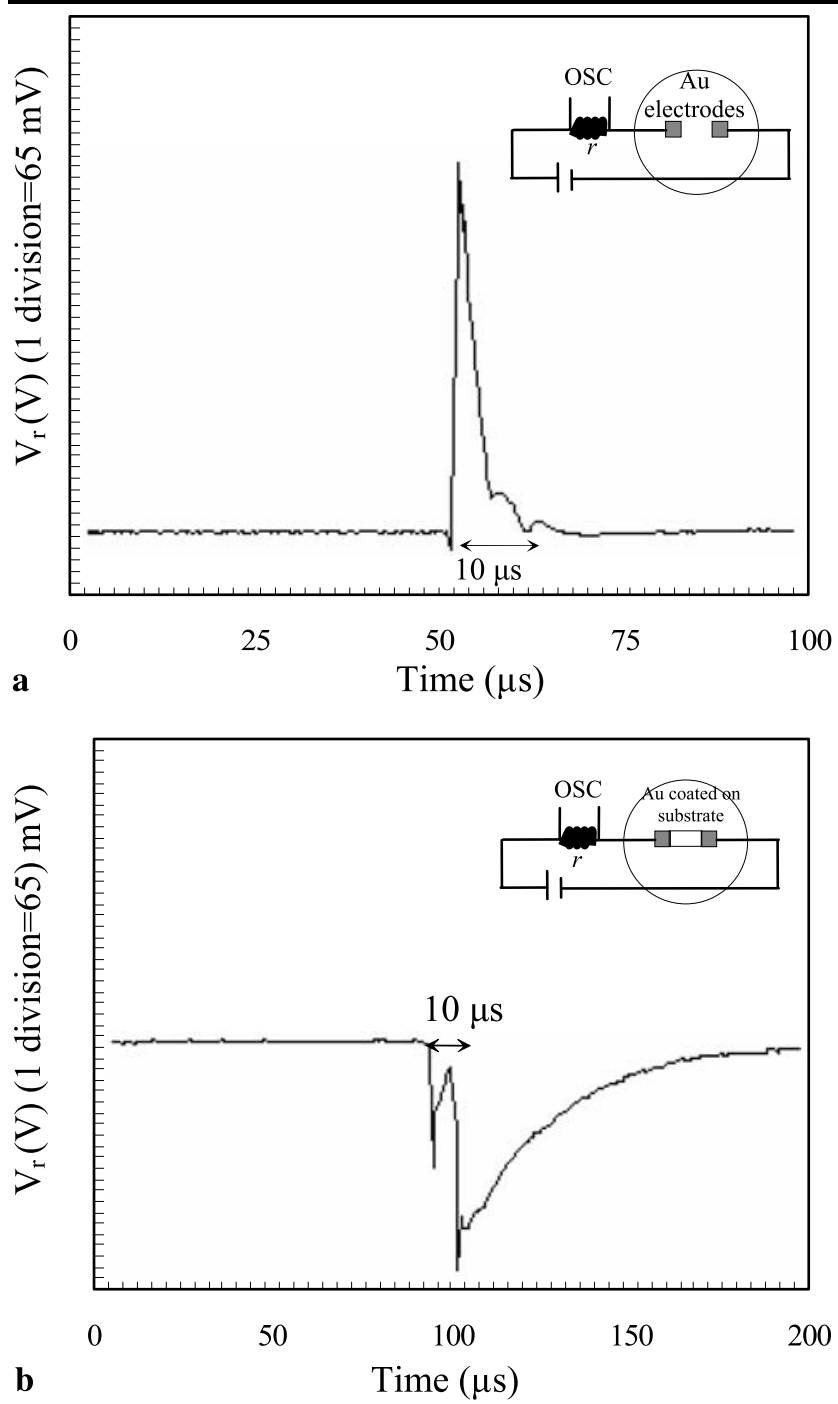

Fig. 6 (a) Voltage signal corresponding to laser plume propagation with time duration of nearly $8 \mu \mathrm{s}$. (b) The voltage signal of reference constant resistance $R$ related to the peak at 1025 pulses

we suggest a surface mechanism assuming the oxygen as the main reason of conductance fluctuations. We suppose that absorption of oxygen takes place during plume-surface interaction, which is accompanied by oxide condensation from the plume species, resulting in a transient reduction of conductance. Then, oxygen atoms escape gradually from the surface to vacuum and the film's conductance recovers its main value.

To justify oxidation and desorption effects, we refer again to the XPS results (Fig. 2) that showed a sub-stoichiometry surface of a $\mathrm{WO}_{x}$ deposit with $x \sim 1$. In fact, enhancement of peak height (stage 2) is due to both decrease in sample conductance (due to oxide layer formation) and increase in contact resistance of the plume-Au path. However, as the film grows the surface has less effect on sample conductance as well as electrical conductance through the incident

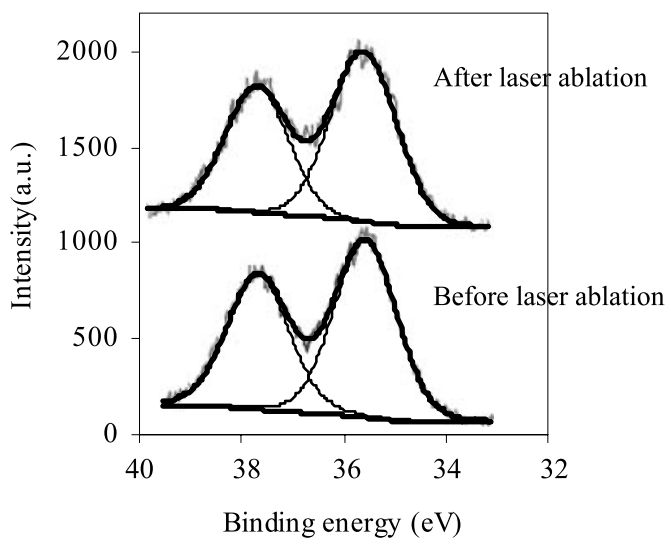

Fig. $7 \mathrm{~W}_{4 f}$ XPS spectra of $\mathrm{WO}_{3}$ target (a) before and (b) after 9000-shot laser irradiation in vacuum

plume; then in the third stage it is expected that the electrical current passes mainly through the bulk and the surface fluctuation gradually vanishes as is shown in Fig. 5.

With regard to the presented data, it seems that a main reason of sub-stoichiometry of tungsten oxide films in the PLD process in vacuum is surface desorption of oxygen from the deposit and fast resistance measurement is capable of monitoring such a process. Also, the distribution order of $\mathrm{W}^{4+}>\mathrm{W}^{5+}>\mathrm{W}^{6+}$ proposes that, in the vacuum condition, oxygen dissociation from tungsten bonds occurs in a typical $\mathrm{W}^{6+} \rightarrow \mathrm{W}^{5+} \rightarrow \mathrm{W}^{4+}$ sequence, which finally leads to a higher concentration of $\mathrm{W}^{4+}$.

\subsection{Target effects}

Laser-target interactions may be another origin of substoichiometry. So, we express here the effect of laser-beam exposure on the target using XPS, XRD and SEM analysis. $\mathrm{W}_{4 f}$ XPS peaks of targets before and also after laser ablation in vacuum are shown in Fig. 7. After deconvolution two characteristic peaks of $\mathrm{W}^{6+}$ are fitted with regard to suitable peak parameters, as already discussed. No considerable change is observed in the spectra. Quantitative analysis indicated that $\mathrm{O} / \mathrm{W}$ remained $\sim 3$ by laser ablation. So, surface states of the target remain nearly the same by laser ablation. However, laser irradiation changes the target color from yellow to dark blue. To clarify this effect we obtained X-ray diffraction spectra of the $\mathrm{WO}_{3}$ target before laser ablation and after 9,000 shots (Fig. 8). The XRD data of targets are shown in Table 2. One can see for both patterns that [001] crystallography orientation has the highest intensity. The calculated lattice parameters are as follows: $a=7.310, b=7.536$ and $c=3.847$ for the target before ablation and $a=7.306, b=7.524$ and $c=3.849$ after ablation. According to JCPDX file No. 20-1323, the $\mathrm{WO}_{3}$ target has a triclinic polycrystalline structure which remains mainly the same after 9000 shots of laser ablation. However, the relative intensity corresponding to different peaks 


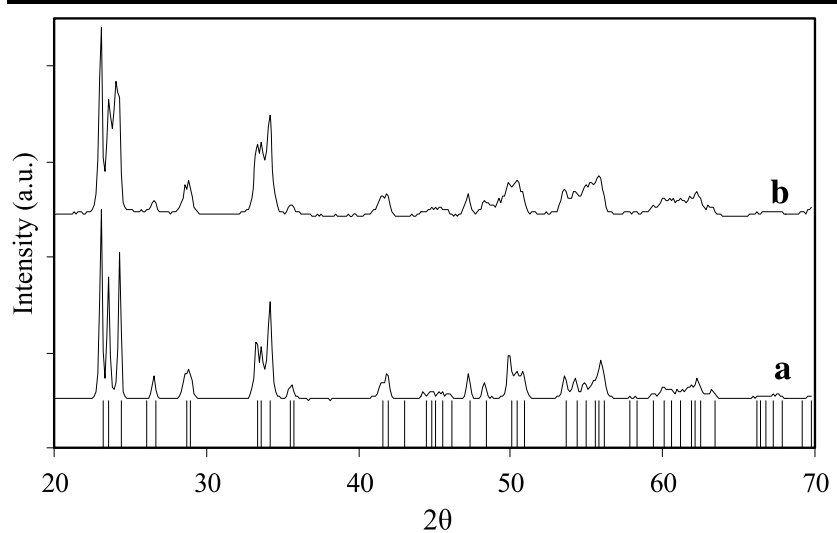

Fig. 8 XRD patterns of $\mathrm{WO}_{3}$ target (a) before and (b) after 9000-shot laser irradiation in vacuum. The reference peaks for triclinic $\mathrm{WO}_{3}$ is shown on the axis

Table 2 XRD data of $\mathrm{WO}_{3}$ target (a) before laser irradiation and (b) after 9000-shot laser irradiation

\begin{tabular}{|c|c|c|c|c|}
\hline & $2 \theta$ & $d_{h k l}(\AA)$ & $\begin{array}{l}\text { Identified } \\
\text { planes }(h k l)\end{array}$ & $\begin{array}{l}\text { Intensity } \\
(\%)\end{array}$ \\
\hline \multirow[t]{10}{*}{ (a) } & 23.15 & 3.847 & 001 & 100 \\
\hline & 23.65 & 3.768 & 020 & 62.4 \\
\hline & 24.39 & 3.655 & 200 & 72.2 \\
\hline & 26.64 & 3.351 & 120 & 12.3 \\
\hline & 28.97 & 3.120 & 111 & 13.7 \\
\hline & 33.29 & 2.696 & 021 & 27.7 \\
\hline & 33.63 & 2.669 & 201 & 22.4 \\
\hline & 34.20 & 2.626 & 220 & 44.6 \\
\hline & 48.40 & 1.884 & 040 & 7.1 \\
\hline & 49.97 & 1.828 & 140 & 24.8 \\
\hline \multirow[t]{10}{*}{ (b) } & 23.15 & 3.849 & 001 & 100 \\
\hline & 23.65 & 3.762 & 020 & 53.6 \\
\hline & 24.40 & 3.653 & 200 & 60 \\
\hline & 26.66 & 3.349 & 120 & 5.6 \\
\hline & 28.95 & 3.089 & 111 & 14.3 \\
\hline & 33.31 & 2.694 & 021 & 32.6 \\
\hline & 33.64 & 2.668 & 201 & 32.6 \\
\hline & 34.22 & 2.624 & 220 & 43.8 \\
\hline & 48.31 & 1.887 & 040 & 5.6 \\
\hline & 49.93 & 1.829 & 140 & 14.3 \\
\hline
\end{tabular}

varies by laser exposure (Table 2). The relative intensities such as [020], [200], [120] and [140] decrease relative to the [001] peak while [111], [021] and [201] increase. We attributed this effect to laser-induced phase changes and it may be accompanied by creation of oxygen defects at the top layer of the target. The surface morphology of the target before and after laser irradiation is also investigated by SEM. In Fig. 9 a SEM micrograph of the $\mathrm{WO}_{3}$ target before

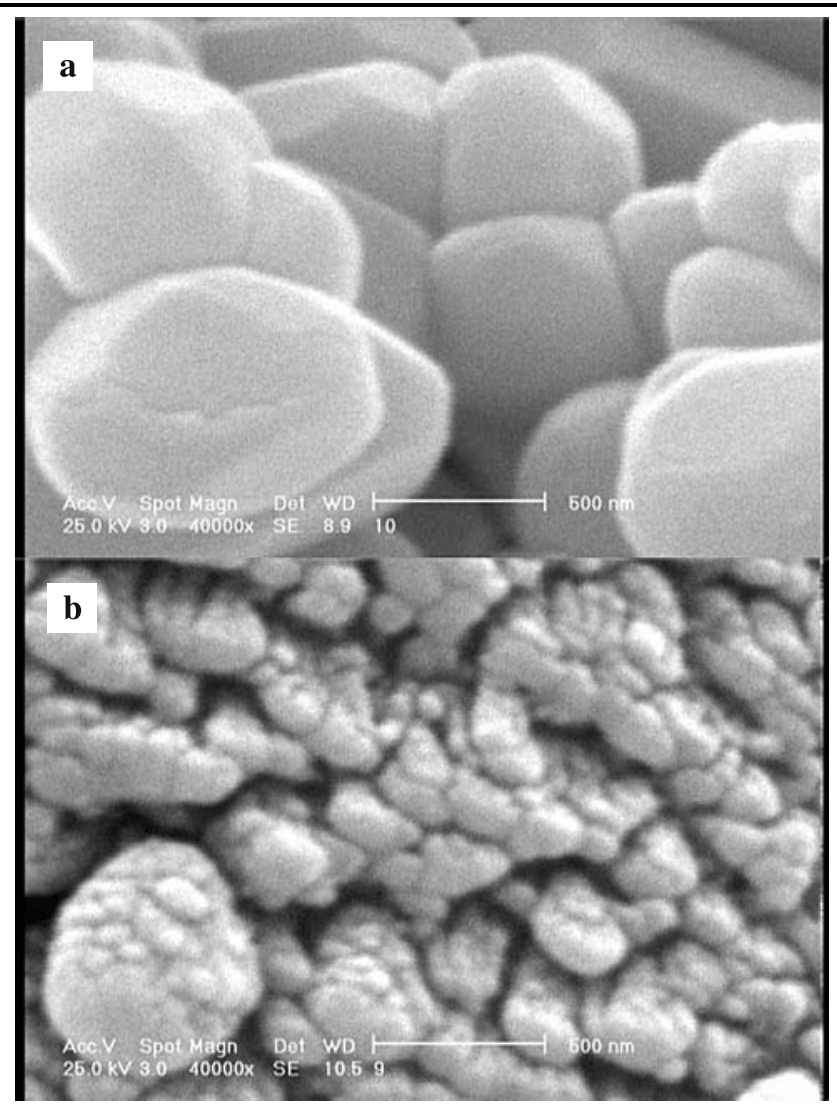

Fig. 9 SEM images of target (a) before and (b) after 9000-shot laser irradiation

laser ablation indicates a polycrystalline structure including micron-size particles, while after laser irradiation the surface morphology was modified to small attachments of particles. Therefore, laser-target interaction mainly changes the structure and morphology but does not affect the oxygen concentration of deposits.

\section{Conclusion}

$\mathrm{WO}_{x}$ thin films were deposited on $\mathrm{SiO}_{2}$ glass or $\mathrm{Si}(111)$ substrates by PLD. The compositions of the films were found to be $\mathrm{WO}_{x \sim 3.1}$ and $\mathrm{WO}_{x \sim 1}$ with different distributions of tungsten chemical states. Electrical resistance measurements during film growth revealed some microsecond fluctuations on the background of the sample resistance curve versus time. At the early stages of film growth we attributed them to conduction through the plume, while near the film percolation stage their intensity reduces and then changes to resistance enhancement peaks. In this stage we attributed such instabilities to formation of an oxide surface layer by received oxygen and $\mathrm{WO}_{x}$ species and subsequently desorption of oxygen from the surface of the sample. The oxidation time is similar to the plume-surface interaction duration; however, desorption has a higher time 
scale. So, we could trace oxygen desorption at the surface by fast electrical measurement during the film's growth. Finally, the effect of the laser beam on the target's structure, morphology and oxygen concentration was studied. Our results revealed that laser-target interaction leads to structure and surface modification while oxygen states of the target remain unchanged.

Acknowledgements The authors wish to thank the Research Council of Sharif University of Technology and the Hi Tech Center for financial support. The authors also wish to thank Dr. M.M. Ahadian for useful comments, Mr. Rafiey at the ESCA/AES laboratory and also Z. Mohammadzadeh for her kind help.

Open Access This article is distributed under the terms of the Creative Commons Attribution Noncommercial License which permits any noncommercial use, distribution, and reproduction in any medium, provided the original author(s) and source are credited.

\section{References}

1. C.G. Granqvist, Handbook of Inorganic Electrochromic Materials (Elsevier, Amsterdam, 1995)

2. A. Rougier, F. Portemer, A. Quede, M. El Marssi, Appl. Surf. Sci. 153, 1 (1999)

3. E. Haro-Poniatowski, M. Jouanne, J.F. Morhange, C. Julien, R. Diamant, M. Fernandez-Guasti, G.A. Fuentes, J.C. Alonso, Appl. Surf. Sci. 127, 674 (1998)
4. G. Soto, W. De La Cruz, J.A. Díaz, R. Machorro, F.F. Castillón, M.H. Farías, Appl. Surf. Sci. 218, 281 (2003)

5. M. Schuisky, J.W. Elam, S.M. George, Appl. Phys. Lett. 81, 180 (2002)

6. G. Fahsold, A. Bartel, O. Krauth, N. Magg, A. Pucci, Phys. Rev. B 15, 14109 (2000)

7. M.S. Tillack, D.W. Blair, S.S. Harilal, Nanotechnology 15, 390 (2004)

8. D.B. Chrisey, G.K. Hubler, Pulsed Laser Deposition of Thin Films (Wiley, New York, 1994)

9. Y.G. Shen, Y.W. Mai, Mater. Sci. Eng. A 284, 176 (2000)

10. I.M. Litas, P. Vinatier, A. Levasseur, J.C. Dupin, D. Gonbeau, F. Weill, Thin Solid Films 416, 1 (2002)

11. M. Occhiuzzi, D. Cordischi, D. Gazzoli, M. Valigi, P.C. Heydorn, Appl. Catal. A: Gen. 269, 169 (2004)

12. F. Di Gregorio, V. Keller, J. Catal. 225, 45 (2004)

13. R.J. Colton, A.M. Guzman, J.W. Rabalais, J. Appl. Phys. 49, 409 (1978)

14. J.R. Regalbuto, T.H. Fleisch, E.E. Wolf, J. Catal. 107, 114 (1987)

15. J.F. Fiedor, A. Proctor, M. Houalla, D.M. Hercules, Surf. Interface Anal. 23, 204 (1995)

16. M. Regragui, M. Addou, A. Outzourhit, J.C. Bernede, E.E. Idrissi, Thin Solid Films 358, 40 (2000)

17. B.V. Crist, Handbook of the Elements and Native Oxides (XPS International, Kawasaki, 1999)

18. G.A. Niklasson, L. Berggern, A.L. Larsson, Sol. Energy Mater. Sol. Cells 84, 315 (2004)

19. B. Ingham, S.C. Hendy, S.V. Chong, J.L. Tallon, Phys. Rev. B 72, 075109 (2005)

20. K. Bange, Sol. Energy Mater. Sol. Cells 58, 1 (1999) 